\title{
Dynamical response function of a compressed lithium monolayer.
}

\author{
A. Rodriguez-Prieto ${ }^{1,2}$, V. M. Silkin ${ }^{1}$, A. Bergara ${ }^{1,2}$, and P. M. Echenique ${ }^{1,3}$ \\ 1 Donostia International Physics Center (DIPC), \\ Paseo de Manuel Lardizabal, 20018, \\ Donostia, Basque Country, Spain \\ 2 Materia Kondentsatuaren Fisika Saila, \\ Zientzia eta Teknologia Fakultatea, \\ Euskal Herriko Unibertsitatea, 644 Postakutxatila, \\ 48080 Bilbo, Basque Country, Spain \\ 3 Materialen Fisika Saila, Kimika Fakultatea, \\ Euskal Herriko Unibertsitatea, 20080, \\ Donostia, Basque Country, Spain
}

\begin{abstract}
Since recent both theoretical and experimental results have proved that the simple behaviour light alkaline metals present at equilibrium breaks when high pressures are applied, they have become an important object of study in Condensed Matter Physics. On the other hand, development of new techniques in the atomic manipulation allows the growth of atomic monolayers (ML's), therefore rising the interest to analyze low dimensional systems under different conditions. In particular, new ab initio calculations performed for a lithium ML show that its electronic properties experience important modifications under pressure, which could lead to significant modifications in its dynamical response function. In this article we perform ab initio calculations of the dynamical response function of a lithium ML analyzing its evolution with increasing applied pressure. We show that besides the well known intraband and interband plasmons, rising electronic density induces characteristic features of acoustic plasmons related to the presence of two types of carriers at the Fermi level.
\end{abstract}

PACS numbers:

\section{INTRODUCTION}

Light alkali metals have lately become an important object of study due to the complexity induced by pressure in their behaviour, in contrast to the simple picture they present under normal conditions of pressure and temperature. At equilibrium, alkalies are frequently considered to be simple metals due to their crystallization in high symmetric structures, monovalency and high conductivity. At the same time the interaction between valence electrons and ionic cores, or pseudopotential, has been argued to be weak in such systems. As a consequence, the nearly free electron (NFE) model accurately describes their electronic properties in absence of applied pressure [1] as it can be easily checked by their almost spherical Fermi surfaces. However, when high pressures are applied, the magnitude of the pseudopotential rises, the core electrons substantially overlap and the otherwise valid NFE model breaks. Neaton et al [2] theoretically analyzed compressed bulk lithium, predicting that high pressures could induce phase transitions to less symmetric, lower coordinated structures, associated to electronic localizations. These theoretical predictions were experimentally confirmed by Hanfland et al 3], who found that lithium undergoes several phase transitions from a simple, high symmetric, $b c c$ structure at equilibrium to a complex $c I 16$ with 16 atoms per unit cell at around $40 \mathrm{GPa}$. It is important to mention that pressure induced transitions from simple to more complex structures are not singular to lithium but have also been observed in heavier alkalines [4]. Another important fea- ture which shows up the complexity induced by pressure in the behaviour of light alkalies is that despite the superconducting transition for lithium at equilibrium has not been found yet, $T_{c}<100 \mu \mathrm{K}[5]$, when compressed to around $30 \mathrm{GPa}, T_{c}$ rises up to $15 \mathrm{~K}$ [6, 7], becoming the highest transition temperature between simple elements [8]. Therefore, the characterization and understanding of the physical properties of compressed light elements becomes a priority. On the other hand, the development of new techniques for the atomic manipulation allows the growth of atomic monolayers (ML's) on inert substratum, semiconductors or noble gases. These new possibilities rise the interest to analyze physical properties of low-dimensional systems under different conditions, as it could be the case of a lithium ML under pressure. In addition, extending our conclusions to the bulk will also give another perspective to understand the physical origin under the experimentally observed features in compressed lithium. Previous ab initio analysis of the structural and electronic properties of a lithium ML 9, 10] reveal important modifications in both its band structure and Fermi line, which will also lead to significant modifications on its dynamical response function, up to now just studied at equilibrium [11]. In this article we perform calculations of the dynamical response function of a lithium ML at different pressures, analyzing its evolution with increasing electronic density. Besides the common intraband and interband plasmons, at a certain value of the lateral pressure applied to the ML, we observe characteristic features of acoustic plasmons related to the presence, at this pressure range, of two types of carriers at the Fermi level. 
In Section III we describe the theoretical and computational background of this work. Results and Discussion are presented at Section Un Unless otherwise stated we use atomic units throughout, i.e., $e^{2}=\hbar=m_{e}=1$.

\section{THEORETICAL AND COMPUTATIONAL BACKGROUND.}

We consider a perturbing positive charge located far from the ML, $z_{0}>d$ ( $d$ being the thickness of the ML). Then, the differential cross section for a process in which the electron is scattered with energy $\omega$ and momentum transfer $|\mathbf{q}|$ is proportional to $\operatorname{Im} g(\mathbf{q}, \omega)$, where $g(\mathbf{q} ; \omega)$ is defined as the density response function of the ML [12, 13],

$$
\begin{gathered}
g(\mathbf{q} ; \omega)=-\frac{2 \pi}{|\mathbf{q}|} \int d z \int d z^{\prime} \times \\
\times \chi_{\mathbf{G}=0, \mathbf{G}^{\prime}=0}\left(\mathbf{q}, z, z^{\prime} ; \omega\right) e^{|\mathbf{q}|\left(z+z^{\prime}\right)},
\end{gathered}
$$

where $\mathbf{q}$ belongs to the two-dimensional Brillouin zone (2DBZ) and $\mathbf{G}$ and $\mathbf{G}^{\prime}$ are reciprocal two-dimensional lattice vectors. In order to obtain $g(\mathbf{q} ; \omega)$ we strictly follow the method presented in Refs. [11, 14]. Thus, our calculations are performed within the framework of time dependent density functional theory (TDDFT), where the random phase approximation (RPA) 15 has been applied. We use a local density approximation (LDA) in order to calculate the total energy and one-particle eigenvalues and eigenvectors of the ground state, which were evaluated with the use of Troullier-Martins Li pseudopotentials [16]. The description of the ML is implemented by a supercell which contains 20 layers of vacuum between ML's in order to minimize interaction between them. Effects of compression were simulated by reducing the lattice parameter. For the evaluation of $\chi_{\mathbf{G}, \mathbf{G}^{\prime}}^{0}$, previous step in the calculation of $\chi_{\mathbf{G}, \mathbf{G}^{\prime}}$, we implement up to $\sim 80$ bands. In addition, we use $\sim 7700$ k-points for the sampling of the 2DBZ.

\section{RESULTS AND DISCUSSION}

According to previous $a b$ initio calculations, the lithium ML adopts an hexagonal (hex) structure at equilibrium, which corresponds to $r_{s}=3.02$ [21]. However, with applying pressure, a phase transition to the more opened square $(s q)$ structure is produced at $r_{s}=2.25$. In Fig. 1 we plot the band structure and density of states (DOS) of a lithium ML corresponding to the hex structure at equilibrium and the $s q$ structure with $r_{s}=2.15$, close to the structural phase transition. We can observe a quasi free electron-like behaviour at equilibrium, where only one band with a parabolic-type dispersion of $s$-character is occupied. In addition, the DOS for occupied states is almost constant, reflecting the quasi two-dimensional character of the ML. However, we can notice important modifications at higher pressures: the first band flattens
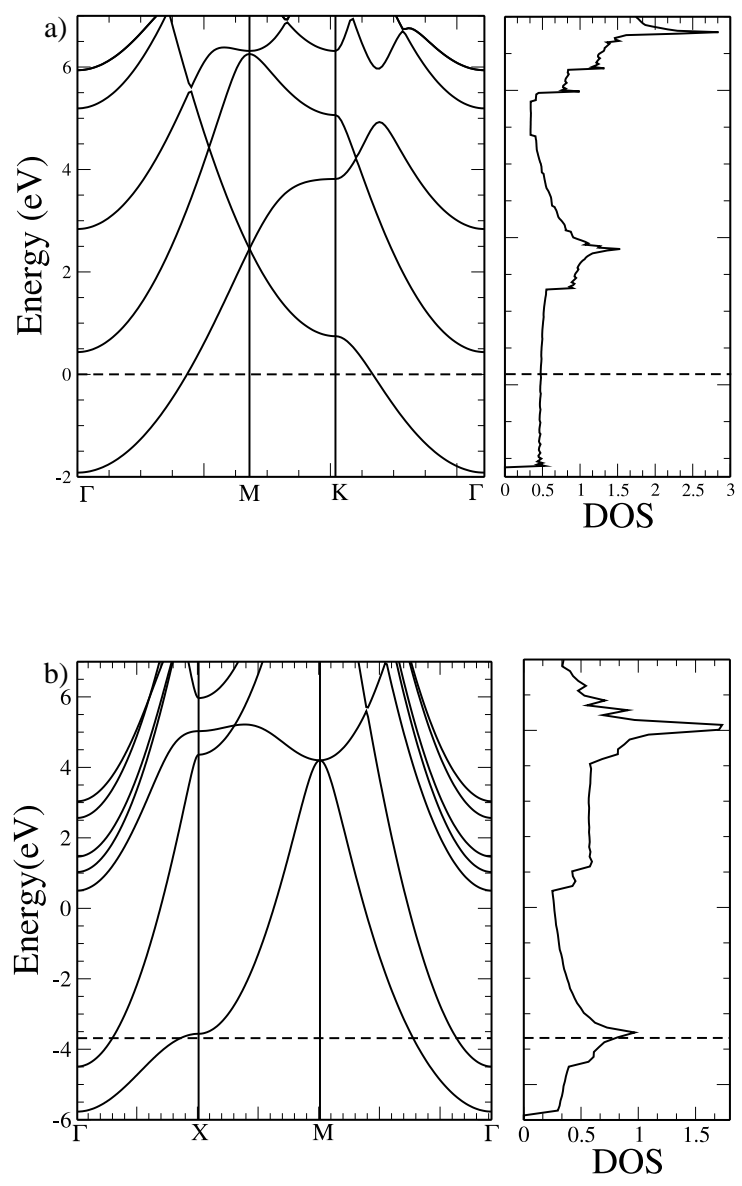

FIG. 1: Band structure and density of states (DOS) for (a) a hexagonal lithium ML at equilibrium ( $r_{s}=3.02$ a.u.) and (b) a square structure at $r_{s}=2.15$ a.u. (close to the structural transition). The Fermi energy is represented by the dotted line. The bandwidth of the first band decreases with pressure and induces the occupation of the second band.

associated to the increasing of the band gap at the zone boundary, indicating a clear electronic localization. At the same time, as a consequence of the decrease in the energy difference between the first two bands at $\Gamma$, the $p_{z}$-band, which is antisymmetric in the direction perpendicular to the plane of the ML, starts to be occupied. Therefore, we have two types of carriers at the Fermi energy, a fact which plays a fundamental role in the pressure induced properties of the electronic collective excitations, as will be explained bellow. It is noteworthy that the Fermi line of the ML also suffers significant deviations with increasing electronic density [10], as it is shown in Fig. 2

These important pressure induced modifications in both the band structure and the Fermi line of the ML are expected to have a profound effect in the dynamical response function. In what follows, we will analyze the loss 


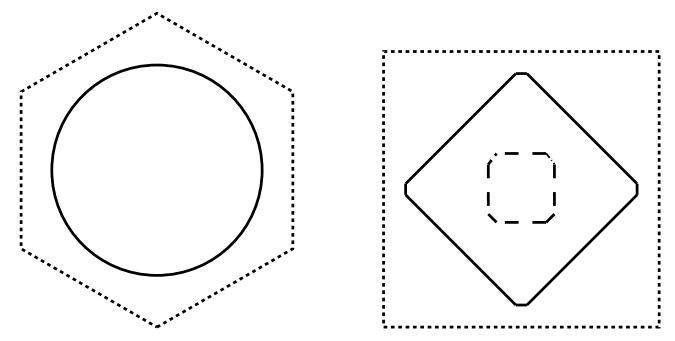

FIG. 2: Fermi lines of a hexagonal lithium ML at equilibrium, $r_{s}=3.02$ (left), and a square lithium ML at $r_{s}=2.15$ (right). Solid line corresponds to the Fermi line of the $s$-band. For values of the density $r_{s} \leq 2.4$, the $p_{z}$-band starts to be occupied, so that this band is represented by a dashed line. The zone boundary is plotted with a dotted line.

function, proportional to the imaginary part of $g(\mathbf{q} ; \omega)$, at two different selected densities, $r_{s}=3.02$ (equilibrium) and $r_{s}=2.4$. At the latter density the phase transition has not yet been produced whereas the $p_{z}$-band is already occupied. In such a way, we can conclude that the physical origin of the interesting phenomena which will be described bellow is not related to the hex $\rightarrow s q$ phase transition, but is a direct consequence of the presence of two different conductors at the Fermi energy. In

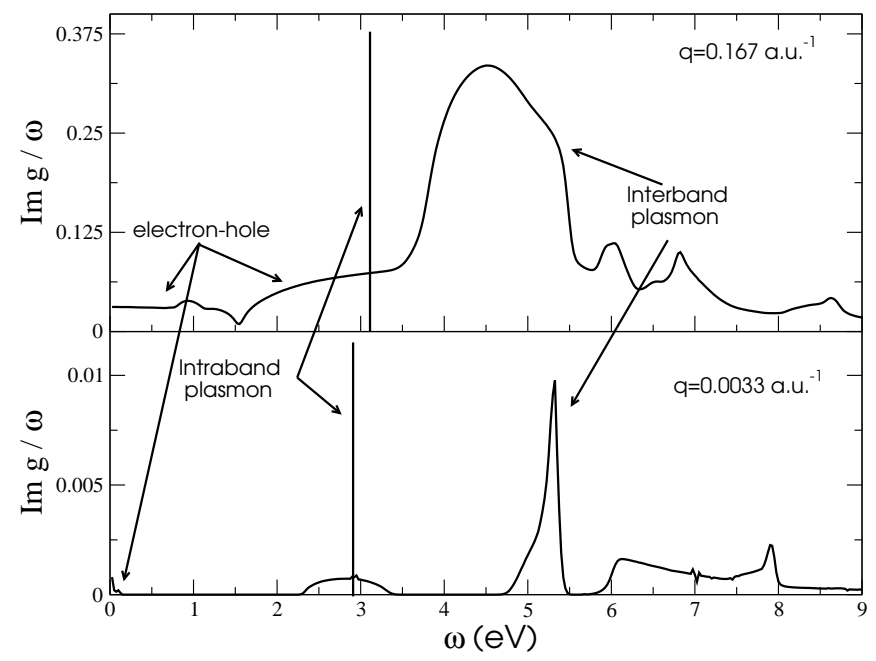

FIG. 3: Loss function $\operatorname{Im} g(\mathbf{q} ; \omega)$ of the hexagonal lithium ML at equilibrium for two different values of the wavefunction in the $\Gamma K$ direction. One can see one-particle electron-hole excitations and two peaks associated to the intraband and interband plasmons.

Fig. 3 we present the loss function of a lithium ML at equilibrium for two selected wavevectors in the $\Gamma K$ direction. As we are mainly interested in collective excitations, small momentum transfers are considered. Our spectra consists in one-particle electron-hole excitations with features corresponding to intraband excitations at small energies and interband excitations at higher energies, together with two plasmon peaks. The first peak is situated at an energy close to $\sim 3 \mathrm{eV}$ and linked to the in- traband collective excitations inside the $s$-band, whereas the second one with energy of $\sim 5 \mathrm{eV}$ is related to the interband plasmon, which physically corresponds to collective motion of electrons in the direction perpendicular to the ML. Dispersion curves for both intraband and inter-

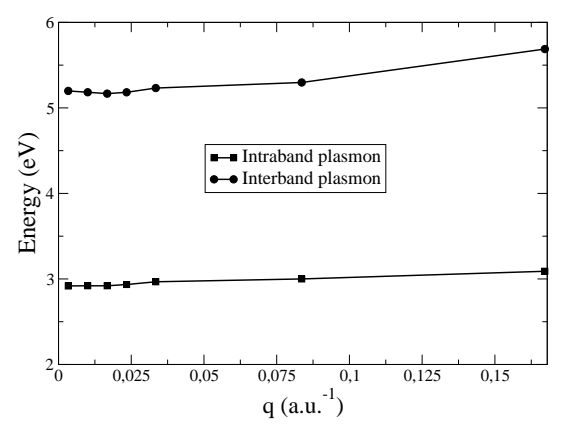

FIG. 4: Dispersion curves of the ab initio collective intraband (squares) and interband (circles) excitations in the $\Gamma K$ direction. The non-zero minimum energy in the limit $\mathbf{q} \rightarrow 0$ indicates that electrons located at neighbouring layers feel the interaction.

band collective excitations are plotted in Fig. 4 As our selected wavefunctions are much smaller than the characteristic Fermi momentum of the ML, we would expect a $\sqrt{\mathbf{q}}$ dispersion [17] for the two-dimensional intraband plasmon, instead of the non-zero minimum energy for 3D plasmons [15]. However, the Fourier transform of the electronic potential of electrons located at neighboring layers decays exponentially with $|\mathbf{q}| L$, so that when dealing with vectors whose size is comparable to the inverse of the intermonolayer distance, $q \simeq 1 / L=0.05 \mathrm{a} . \mathrm{u}$, electrons feel the interaction with other layers. Therefore, the plasmons show a three dimensional character at very small momentum, which is reflected in the gap at $|\mathbf{q}| \rightarrow 0$ that can be seen in Fig. 4.

Loss function of the ML with $r_{s}=2.4$ at selected momenta in the $\Gamma K$ direction is presented in Fig. 5] The $s$-bandwidth at this density is very similar to that at equilibrium. As a consequence, the collective excitations inside the $s$-band require a very similar energy at both densities, equilibrium and $r_{s}=2.4$. Thus, the intraband plasmon peak is situated at $\sim 3.5 \mathrm{eV}$, close to the location at equilibrium. However, the energy gap between the first two bands, $s$ and $p_{z}$, increases under pressure, therefore requiring more energy the collective motions of electrons between these two bands to be excitated, and shifting the corresponding interband plasmon peak to around $\sim 6.5$ $\mathrm{eV}$. As well as the two plasmon peaks appearing also in absence of applied pressure, here one can also observe peaks associated with two other interband collective excitations with energies $\sim 7.7 \mathrm{eV}$ and $\sim 9.5 \mathrm{eV}$, due to the presence of two partly occupied energy bands. In addition, a remarkable peak is induced by pressure at energies close to zero. Besides the common intraband and interband collective excitations, the presence of two type of 


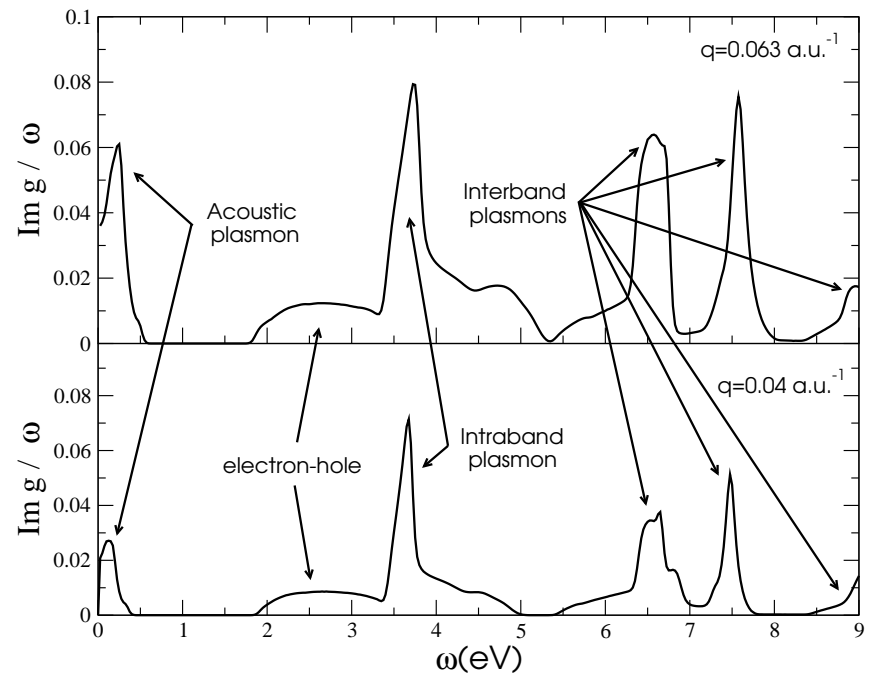

FIG. 5: Loss function $\operatorname{Im} g(\mathbf{q} ; \omega)$ of the hexagonal lithium ML with $r_{s}=2.4$ for two different values of the wavefunction in the $\Gamma K$ direction. Besides the common intraband plasmon, close to the same energy as at equilibrium, and interband one, located at a higher energy, one can also notice a remarkable peak at small $\omega$, the characteristic feature of an acoustic plasmon.

carriers at the Fermi level with different velocities allows the existence of another kind of collective modes, known as acoustic plasmons because of their linear dispersion in |q| 18], as it can be checked in Fig. [6] The same acoustic plasmon was also predicted for a berilium ML [11]. Contrary to the case of two dimensional-like plasmons, which are well established experimentally [19, 20], the acoustic counterparts have not yet been unambiguously identified. It is known that, in this case, the light electrons screen the Coulomb repulsion between the electrons with a lower velocity, so that an additional intraband acoustic collective excitation is produced.

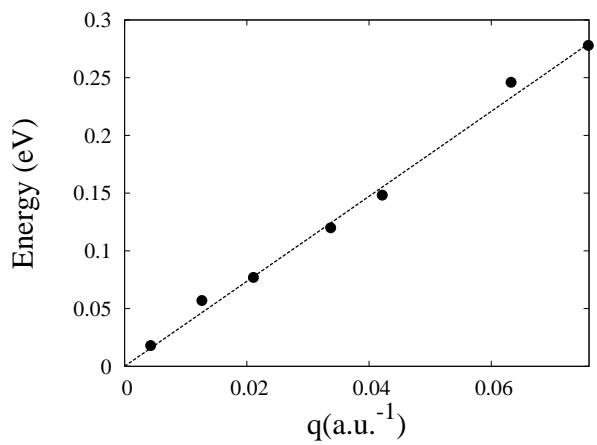

FIG. 6: Dispersion curve of the $a b$ initio acoustic plasmon induced by the applied pressure in a hexagonal lithium ML with $r_{s}=2.4$. A clear lineal dependence with $|\mathbf{q}|$ can be observed. Dots are calculations whereas dashed line is a fit. The slope of the fit is close to the velocity of the slow electrons.
[1] E. Wigner and F. Seitz, Phys. Rev. 43 (1933) 804.

[2] J. B. Neaton and N. W. Ashcroft, Nature 400 (1999) 141.

[3] M. Hanfland, K. Syassen, N. E. Christensen and D. L. Novikov, Nature 408 (2000) 174.

[4] J. B. Neaton and N. W. Ashcroft, Phys. Rev. Lett. 86 (2001) 2830.

[5] K. I. Juntunen and J. T. Tuoriniemi, Phys. Rev. Lett. 93 (2004) 157201.

[6] K. Shimizu, H. Ishikawa, D. Takao, T. Yagi and K. Amaya, Nature 597 (2002) 597.

[7] S. Deemyad and J. S. Shilling, Phys. Rev. Lett. 91 (2003) 167001.

[8] N. W. Ashcroft, Nature 419 (2002) 569.

[9] A. Bergara, J. B. Neaton and N. W. Ashcroft, Phys. Rev. B 62 (2000) 8494.

[10] A. Rodriguez-Prieto and A. Bergara, Phys. Rev. B 72 (2005) 125406.

[11] A. Bergara, V. M. Silkin, E. V. Chulkov and P. M. Echenique, Phys. Rev. B 67 (2003) 245402.

[12] B. N. J. Persson and E. Zaremba, Phys. Rev. B 31 (1985) 1863.
[13] K. D. Tsuei, E. W. Plummer, A. Liebsch, E. Pehlke, K. Kempa and P. Bakshi, Surf. Sci. 302 (1991) 247.

[14] V. M. Silkin, E. V. Chulkov and P. M. Echenique, Phys. Rev. Lett. 93 (2004) 176801.

[15] See, e.g., D. Pines, Elementary Excitations in Solids, Addison-Wesley, New York, 1963.

[16] N. Troullier and J. L. Martins, Phys. Rev. B 43 (1991) 1993.

[17] F. Stern, Phys. Rev. Lett. 18 (1967) 546.

[18] V. M. Silkin, A. García-Lekue, J. M. Pitarke, E. V. Chulkov, E. Zaremba and P. M. Echenique, Europhys. Lett. 66 (2004) 260.

[19] T. Nagao, T. Hildebrant, M. Henzler and S. Hasegawa, Surf. Sci. 493 (2001) 680.

[20] T. Nagao, T. Hildebrant, M. Henzler and S. Hasegawa, Phys. Rev. Lett. 86 (2001) 5747.

[21] $r_{s}$ is the two-dimensional linear density parameter, defined by the relation $A / N=\pi\left(r_{s} a_{0}\right)^{2}$ where $N$ is the number of nuclei in a ML with area $A$ and $a_{0}$ is the Bohr radius. 